\title{
A plan quality classifier derived with overlap-wall-histogram of hollow organs for automatic IMRT plan quality control of prostate cancer cases
}

\author{
Ting Song, Zhen Tian, Xun Jia, Linghong Zhou, Steve B Jiang, Xuejun Gu \\ Department of Radiation Oncology, UT Southwestern Medical Center, Dallas, TX, USA.
}

Received March 19, 2014; Published Online April 08, 2014

[Presented at the Young Investigator's Symposium at the 2014 Annual Meeting of

Southwest Chapter of American Association of Physicists in Medicine (AAPM) in San Antonio, Texas, USA]

\section{Conference Proceeding}

\begin{abstract}
Purpose: We developed a plan quality classification model to assess IMRT plan quality of prostate cancer patients for automatic plan quality control.

Methods: For hollow organs such as rectum and bladder, dose-wall-histogram (DWH) was used to evaluate OAR dose sparing in our institution. Correspondingly, we proposed a new descriptor called overlap-wall-histogram $(\mathrm{OWH})$ to describe the complex spatial relationship between PTV and a hollow organ. Two metrics calculated from the OWH and DWH are introduced to quantitatively evaluate the difficulty of patient geometry for planning and plan quality in terms of OAR sparing, respectively. A linear correlation between these two metrics was observed after plotting plan quality metric as a function of geometry difficulty metric studied from a database of prostate cases treated in our institution with acceptable plan quality. Thus, a fitting line was built acting as the boundary of high quality and poor quality plans. A query plan falling above the boundary is assessed as high quality, vice versa poor quality.
\end{abstract}

Results: 15 prostate IMRT plans were used to test our model. One was identified as poor quality and the others were common-level. After re-planning all plans, the dose constraints for bladder wall W75 (percentage of wall receiving more than $75 \mathrm{~Gy}$ ), $\mathrm{W}_{70}, \mathrm{~W}_{65}$ and $\mathrm{W}_{60}$ can be reduced by $3.34 \%$, 3\%, 6.99\%, $6.54 \%$ for that poor quality plan and $1.11 \%, 0.95 \%, 1.45 \%$ and $1.81 \%$ averagely for the com-

Presenting author: Ting Song; Department of Radiation Oncology, UT Southwestern Medical Center, Dallas, TX, USA.

Cite this article as:

Song T, Tian Z, Jia X, Zhou L, Jiang SB, Gu X. A plan quality classifier derived with overlap-wall-histogram of hollow organs for automatic IMRT plan quality control of prostate cancer cases. Int J Cancer Ther Oncol 2014; 2(2):020241.

DOI: $10.14319 /$ ijcto.0202.41 mon-level quality group, without sacrificing PTV coverage and rectum dose sparing.

Conclusion: An effective model was built to provide automatic IMRT plan quality control by evaluating hollow OAR dose sparing for prostate cancer patients. Furthermore, for the query plan with poor quality, potential improvement of plan quality can be estimated and a good reference plan with similar or harder geometry can be automatically chosen from our database to help guide the re-planning if necessary.

Innovation/Impact: We regard this work as innovative because it proposed a new descriptor called overlap-wall- histogram (OWH) (Figure 1) to describe the spatial relationship between PTV and a hollow organ, and two metrics to qualitatively evaluate patient geometry difficulty for planning and plan quality in terms of OAR sparing. Besides, a model was built to establish the boundary of poor quality and high quality plans (e.g. the blue lines in Figure 2) after studying from a training database of prostate cancer IMRT cases with acceptable plan quality. Thus contributes a plan quality classifier to assess IMRT plan quality of prostate cancer patients for automatic plan quality control. A query plan falling above the boundary is assessed as high quality, vice versa poor quality. Furthermore, for a query plan with poor quality, potential improvement of plan quality can be estimated and a good reference plan with similar or harder geometry can be automatically chosen from our database to help guide the re-planning if necessary. 
Key Results: 15 prostate IMRT plans were used to test our model. Their distribution in our IMRT plan quality map is shown in Figure2. One was identified as poor quality and the others were common-level. After re-planning all plans, the dose constraints for bladder wall $\mathrm{W}_{75}$ (the percentage of wall receiving more than

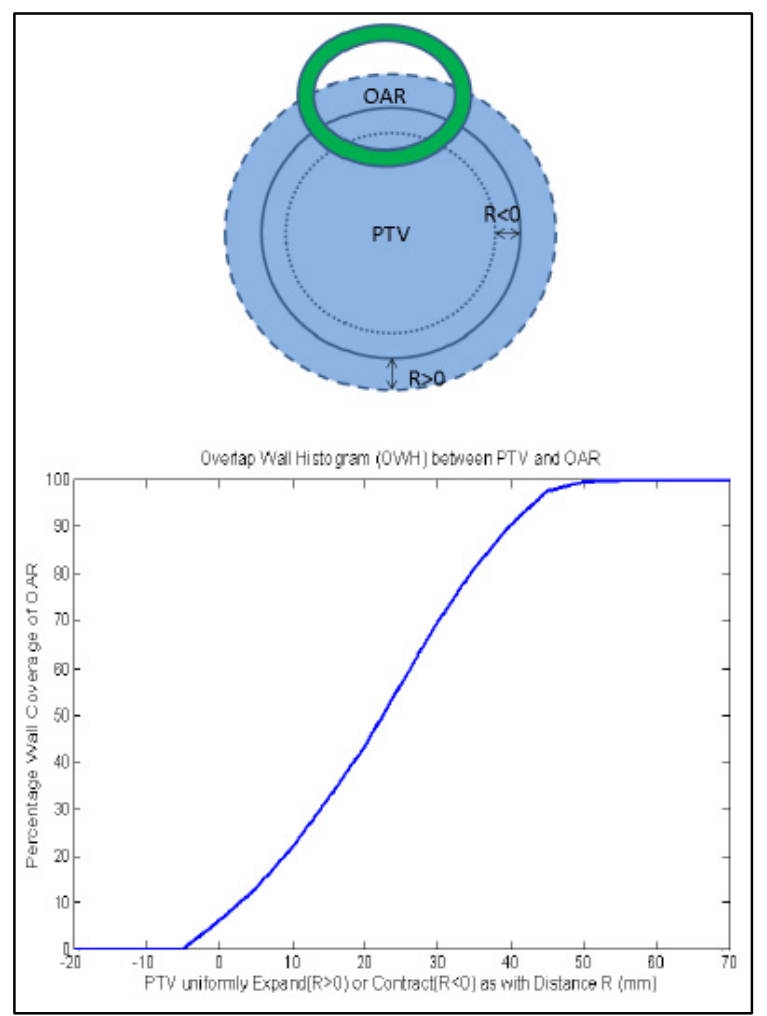

FIG.1: An OWH example. Shape of a target and OAR (above). OWH (below)

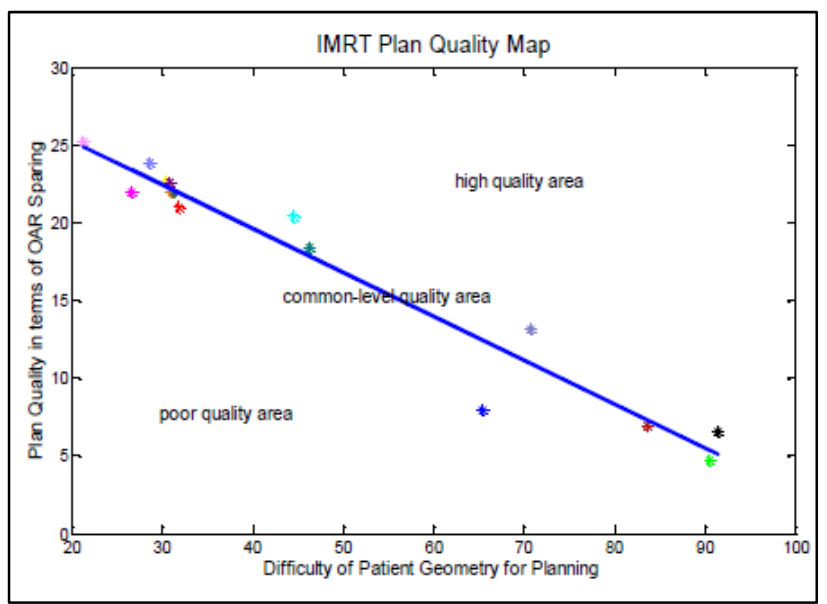

FIG. 2: Established boundary of poor quality and high quality plans studied from a training database
75Gy), $\mathrm{W}_{70}, \mathrm{~W}_{65}$ and $\mathrm{W}_{60}$ can be reduced by $3.34 \%, 3 \%$, $6.99 \%, 6.54 \%$ for that poor quality plan and $1.11 \%$, $0.95 \%, 1.45 \%$ and $1.81 \%$ averagely for the common-level quality group, without sacrificing PTV coverage and rectum dose sparing(Figure 3 ).

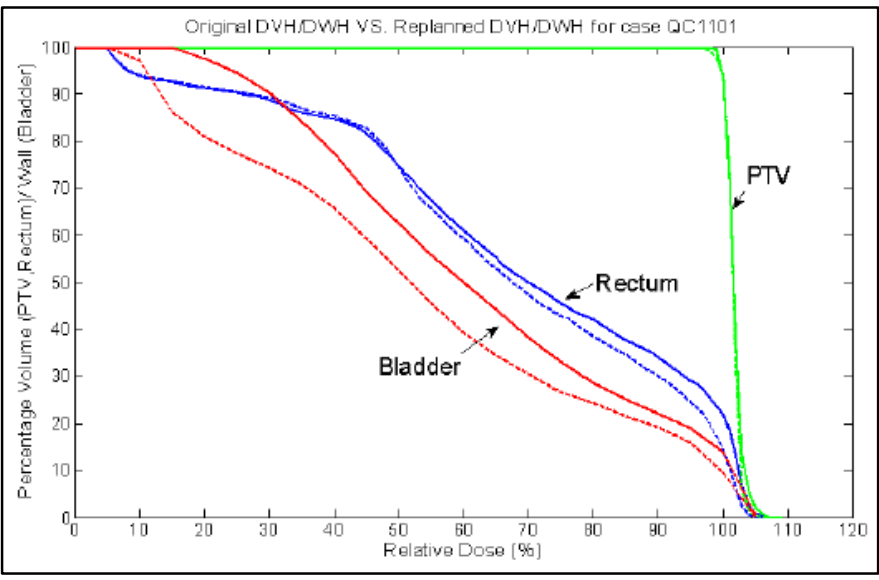

FIG. 3: An example of re-planning improvement for a poor quality plan (original plan- solid line, after re-planning- dashed line) 